\title{
Cardiovascular comorbidities in hospitalised COPD patients: a determinant of future risk?
}

\author{
To the Editor:
}

Cardiovascular disease (CVD), diabetes mellitus and arterial hypertension increase the risk of death and hospitalisations of chronic obstructive pulmonary disease (COPD) patients [1]. COPD patients with CVD are at increased risk of COPD-related hospitalisations [2]. Arterial hypertension is one of the most prevalent comorbidities, influencing $40-60 \%$ of COPD patients [1]. Diabetes mellitus is more prevalent in moderate to very severe COPD than in the general population [1] and hyperglycaemia during acute exacerbations of COPD (AECOPD) is associated with increased in-hospital mortality [3]. The Global Initiative for Chronic Obstructive Lung Disease (GOLD) has proposed a multidimensional classification for COPD management $[4,5]$ that includes symptoms and future risk of AECOPD, based on the severity of airflow limitation and previous exacerbation history. A recent study has shown that the risk of future hospital admission due to COPD and cardiovascular death is higher in the more symptomatic group B compared with group C, regardless the functional advantage of patients in the first group [6]. Our study assessed the effect of CVD, arterial hypertension and diabetes mellitus on the time to first AECOPD, and on exacerbation and hospitalisation risk in groups A-D of the GOLD 2011 and 2013 classification, in a cohort of patients admitted to hospital for AECOPD.

We prospectively enrolled 609 consecutive patients admitted to respiratory medicine departments of two tertiary hospitals with a diagnosis of AECOPD between March 2009 and February 2013. All subjects were current or ex-smokers with $\geqslant 20$ pack-years and a previous spirometry-confirmed diagnosis of COPD. Patients with other comorbid respiratory conditions, comorbidities that might result in a limited expected survival (such as malignancies, leukaemia or AIDS) or with an inability to cooperate with the investigators were excluded. The study protocol was approved by the ethics committees of both hospitals and participants provided informed consent. Symptoms were evaluated according to the COPD assessment test (CAT) [7] and the modified Medical Research Council (mMRC) dyspnoea scale [8]. Spirometry data were from patients' medical records when their COPD was stable (during the last 6 months and $\geqslant 4$ weeks before admission). Patients were categorised into the GOLD 2011 groups, as follows. Group A: fewer symptoms (mMRC $<2 / \mathrm{CAT}<10$ ), forced expiratory volume in $1 \mathrm{~s}\left(\mathrm{FEV}_{1}\right)>50 \%$ predicted and fewer than two exacerbations in the previous year. Group B: more symptoms (mMRC $\geqslant 2 / \mathrm{CAT} \geqslant 10)$, FEV $1>50 \%$ predicted and fewer than two exacerbations in the previous year. Group C: fewer symptoms (mMRC $<2 /$ $\mathrm{CAT}<10), \mathrm{FEV} 1 \leqslant 50 \%$ predicted and/or two or more exacerbations in the previous year. Group D: more symptoms $(\mathrm{mMRC} \geqslant 2 / \mathrm{CAT} \geqslant 10), \mathrm{FEV}_{1} \leqslant 50 \%$ predicted and/or two or more exacerbations in the previous year [4]. In cases of discrepancy between the CAT and MMRC scales, the patient was categorised in the higher category (B or D). We performed an additional analysis classifying all our hospitalised patients in groups C and D based on GOLD 2013, which considers patients with one or more COPD hospitalisation as high risk [5]. CVD, arterial hypertension and diabetes mellitus were prospectively recorded from patients' records, based on the 10th revision of the International Classification of Diseases. CVD was defined as a history of coronary artery disease, chronic heart failure, stroke or arrhythmia [9]. Demographic characteristics of the study subjects are shown in table 1. Patients were followed-up for 1 year with monthly telephone interviews in which they were asked about respiratory symptom deterioration that required treatment with corticosteroids and/or antibiotics or caused any visit to their doctor or to the emergency department, or required hospital admission. The influence of CVD, arterial hypertension and diabetes mellitus on AECOPD and hospitalisations in 1 year was evaluated with multivariate Poisson regression analyses, including significant confounders identified in univariate analyses. Significant confounders in the Poisson regression analysis were age, mMRC dyspnoea score, frequent exacerbator phenotype, $\mathrm{FEV}_{1} \%$ predicted and each one of the three comorbidities examined. Results are presented as incidence rate ratios (IRR) with $95 \%$ confidence intervals. A p-value $<0.05$ was considered statistically significant.

Patients were classified into groups A (23.3\%), B (9.7\%), C (22.0\%) and D (45.0\%). The prevalence of comorbidities increased with increasing disease severity from groups A to D (Chi-squared for trend between GOLD 2011 categories, $\mathrm{p}<0.001$ for all three comorbidities). Exacerbation rates (mean AECOPD per patient per year) increased from 1.5 to $2.4,2.3$ and 3.7 in groups $A-D$, respectively $(\mathrm{p}<0.001)$. The rate of AECOPD in the whole group increased with the presence of comorbidities, from 0.4 in patients with no 
TABLE 1 Demographic and functional characteristics of the study population based on the Global Initiative for Chronic Obstructive Lung Disease 2011 strategy

\begin{tabular}{|c|c|c|c|c|c|c|}
\hline Characteristics & All & Group A & Group B & Group C & Group D & p-value \\
\hline Patients n & 609 & 142 & 59 & 134 & 274 & \\
\hline Males & 557 (91.5) & $130(91.5)$ & $52(88.1)$ & 124 (92.5) & $251(91.6)$ & 0.801 \\
\hline BMI $\mathrm{kg} \cdot \mathrm{m}^{-2}$ & $26.5(24.1-31.3)$ & $28.8(25.4-30.5)$ & $25.8(24.9-29.3)$ & $28.4(24.6-31.3)$ & $25.2(21.6-31.9)$ & 0.002 \\
\hline Current smokers & $163(26.8)$ & $42(29.6)$ & 19 (32.2) & $40(29.9)$ & $62(22.6)$ & 0.089 \\
\hline Smoking history pack-years & $60.0(50.0-90.0)$ & $60.0(50.0-80.0)$ & $75.0(56.0-100.0)$ & $70.0(55.0-92.5)$ & $60.0(50.0-82.5)$ & 0.014 \\
\hline CVD & $331(54.4)$ & 45 (31.7) & $19(32.2)$ & 65 (48.5) & $202(73.7)$ & $<0.001$ \\
\hline DM & 195 (32.0) & 14 (9.9) & $21(35.6)$ & $31(23.1)$ & $127(47.1)$ & $<0.001$ \\
\hline $\mathbf{A H}$ & 410 (67.3) & $79(55.6)$ & $28(47.5)$ & 99 (73.9) & $204(74.5)$ & $<0.001$ \\
\hline Patients with AECOPD ${ }^{\#}$ & $472(77.5)$ & 87 (61.3) & 35 (59.3) & $106(79.1)$ & $244(89.1)$ & $<0.001$ \\
\hline AECOPD per patient per year ${ }^{\#}$ & $2.0(1.0-3.0)$ & $1.0(0.0-1.0)$ & $2.0(0.0-3.0)$ & $2.0(1.0-3.0)$ & $3.0(2.0-5.0)$ & $<0.001$ \\
\hline Patients with hospitalisations" & $290(47.6)$ & $34(23.9)$ & $22.0(37.3)$ & $53.0(39.6)$ & $181(66.1)$ & $<0.001$ \\
\hline
\end{tabular}

Data are presented as $\mathrm{n}(\%)$, mean \pm SD or median (interquartile range), unless otherwise stated. BMI: body mass index; FEV 1 : forced expiratory volume in $1 \mathrm{~s}$; CVD: cardiovascular disease; $\mathrm{DM}$ : diabetes mellitus; $\mathrm{AH}$ : arterial hypertension; AECOPD: acute exacerbations of chronic obstructive pulmonary disease. " : events in the 1-year follow-up. Bold indicates statistical significance.

comorbidities to $1.9,3.6$ and 4.6 AECOPD per patient per year in patients with one, two and three comorbidities, respectively $(\mathrm{p}<0.001)$. Hospitalisation rates in the 1 -year follow-up increased with the number of comorbidities, from 0.1 in patients with no comorbidities to $0.7,1.6$ and 2.0 hospitalizations per patient per year in patients with one, two and three comorbidities, respectively $(p<0.001)$.

Patients with any comorbidity presented shorter times to their first AECOPD compared with those with no comorbidity (log-rank test, $\mathrm{p}<0.001$ ). Patients with increasing number of comorbidities had shorter times to first AECOPD (log rank tests, no one, two and three, and one versus two and three comorbidities, $\mathrm{p}<0.001$ for all pairwise comparisons); no difference between patients with two and three comorbidities was observed $(\mathrm{p}=0.506)$ (fig. 1a). Time to the first AECOPD differed significantly among GOLD 2011 groups (overall comparison $\mathrm{p}<0.001$, log-rank test); there were no differences between groups $\mathrm{A}$ and $\mathrm{B}$, but these patients differed significantly from the high-risk groups $\mathrm{C}$ and $\mathrm{D}$.

In the presence of CVD, the less symptomatic patients (groups $\mathrm{A}$ and $\mathrm{C}$ ) presented more favourable prognosis than the more symptomatic patients of groups $B$ and $D(p<0.001)$ (fig. $1 b)$. A similar pattern was observed in the presence of arterial hypertension $(p<0.001)$ (fig. 1c). In the presence of diabetes mellitus, all patients presented very short times to first AECOPD, with statistical differences being present only in groups $\mathrm{B}$ versus $\mathrm{C}$ and $\mathrm{C}$ versus $\mathrm{D}$ ( $\mathrm{p}=0.009$ and $\mathrm{p}=0.016$, respectively) (fig. $1 \mathrm{~d}$ ). In multivariate Poisson regression analysis, CVD, arterial hypertension and diabetes mellitus were independent predictors of both AECOPD (adjusted IRR (95\% CI) 1.254 (1.117-1.409), 1.960 (1.713-2.242) and 1.389 (1.255-1.538), respectively) and hospitalisations (adjusted IRR (95\% CI) 1.292 (1.075-1.551), 1.798 (1.463-2.211) and 1.489 (1.271-1.745), respectively).

In an additional analysis, based on the fact that hospitalised patients are considered as high risk according to GOLD 2013, all our patients were reclassified in groups C and D. Group C patients presented fewer comorbidities than Group D (median (interquartile range) 1 (0.25-2) versus 2 (1-3), respectively; $\mathrm{p}<0.001)$. Mean AECOPD per patient per year were 1.8 for group $\mathrm{C}$ and 3.5 for group $\mathrm{D}$. Patients with CVD presented higher risk of AECOPD (fig. 1e) and hospitalisations compared with patients without CVD in both C and D Groups ( $\mathrm{p}<0.05$ for all comparisons). Similar results were observed for arterial hypertension and diabetes mellitus. The high 1-year exacerbation rates in our study could be also explained by the fact that previous exacerbations are predictors of future exacerbations [10].

The association of respiratory diseases with CVD, arterial hypertension and diabetes mellitus has been previously described $[11,12]$. To our knowledge, this is the first study that evaluates the impact of these comorbidities in addition to the GOLD 2011/2013 classifications. Possible explanations for this association could be the underlying systemic inflammation and the presence of common risk factors, including cigarette smoking [13]. In our study, we have shown that patients with diabetes mellitus experienced more AECOPD regardless of their COPD category. Recent evidence suggests that COPD patients with diabetes mellitus often 

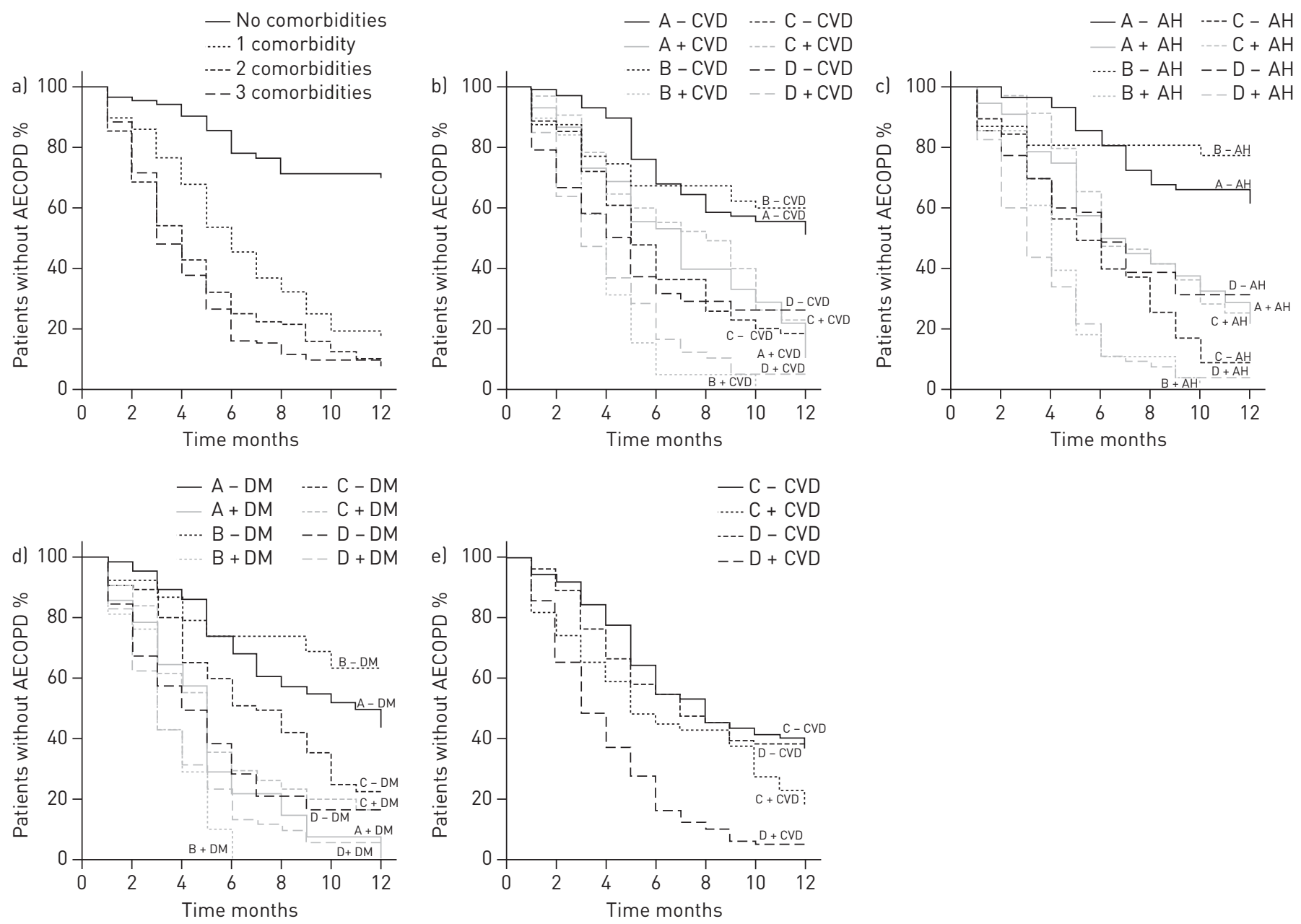

FIGURE 1 Kaplan-Meier curves of the time to first acute exacerbation of chronic obstructive pulmonary disease (AECOPD) according to the presence or absence of comorbidities in the Global Initiative for Chronic Obstructive Lung Disease (GOLD) 2011 groups: a) no comorbidity versus one, two or three comorbidities (cardiovascular disease (CVD), arterial hypertension (AH) or diabetes mellitus (DM)); b) GOLD groups A-D according to the presence or absence of CVD; c) GOLD groups A-D according to the presence or absence of AH; d) GOLD groups A-D according to the presence or absence of DM. Comparisons were performed with log-rank tests. e) Kaplan-Meier curves of the time to first AECOPD according to the presence or absence of CVD in the GOLD 2013 groups.

change from being infrequent to frequent exacerbators [14]. Diabetes mellitus is associated with a poorer immune response, which leads to a higher risk of infections that represent the main cause of AECOPD [15].

During the natural history of COPD, patients who are hospitalised for AECOPD present a less favourable prognosis and AECOPD become more frequent over time in certain patients [16]. Recent studies suggested that the GOLD 2011 COPD classification did not differ from the old spirometric classification in terms of future hospitalisations and mortality [17], and no parameter could predict the change in exacerbation frequency category [14]. The association of the presence of comorbidities with an increased exacerbation risk that is highlighted in our study may represent an important parameter in the evaluation of future risk of COPD patients. This may even be more important in the "low-risk" symptomatic patients with preserved airflow obstruction of group B, who present a higher future risk of AECOPD and/or hospitalisation in the presence of comorbidities.

This study has several limitations. First, the diagnosis of comorbidities was based on patients' histories and medical files, and specific diagnostic procedures were performed in a minority of cases according to doctors' judgment, which may have resulted in underdiagnosis of some comorbidities. A second limitation might be the grouping of several different CVDs, such as heart failure, coronary artery disease and stroke, when at the same time, hypertension (also a cardiovascular abnormality) was evaluated separately. Finally, despite the fact that every effort was made to capture the diagnosis of AECOPD accurately, we cannot exclude that exacerbations of respiratory symptoms can represent worsening of comorbidities, like acute 
heart failure or symptomatic ischaemic heart disease [18]. Further studies are needed to clarify how we can effectively differentiate such events from AECOPD in clinical practice.

In conclusion, in this prospective study, we have shown that the presence of CVD, arterial hypertension and diabetes mellitus in patients hospitalised for AECOPD is associated with a higher risk of exacerbations and hospitalisations for COPD in the following year, especially in the more symptomatic patients of GOLD 2011 groups B and D. Comorbid diseases are independent determinants of future risk in COPD patients, besides the GOLD group, and should be considered in the identification of exacerbation-susceptible patients who may need more intense intervention.

@ERSpublications

Comorbidities are important determinants of future risk in COPD patients and should be considered in the assessment of future risk http://ow.ly/L2qJp

Andriana I. Papaioannou ${ }^{1}$, Konstantinos Bartziokas ${ }^{2}$, Stelios Loukides $^{3}$, Stamatoula Tsikrika ${ }^{1}$, Foteini Karakontaki ${ }^{1}$, Aikaterini Haniotou ${ }^{2}$, Spyros Papiris ${ }^{3}$, Daiana Stolz ${ }^{4}$ and Konstantinos Kostikas ${ }^{3}$

${ }^{1}$ 3rd Respiratory Medicine Dept, Sismanogleion General Hospital, Athens, Greece. ${ }^{2}$ Respiratory Medicine Dept, Amalia Fleming Hospital, Athens, Greece. ${ }^{3}$ 2nd Respiratory Medicine Dept, University of Athens Medical School, Athens, Greece. ${ }^{4}$ Respiratory Medicine Dept, University Hospital, Basel, Switzerland.

Correspondence: Konstantinos Kostikas, 2nd Respiratory Medicine Department, University of Athens Medical School, Rimini 1, 12462 Athens, Greece. E-mail: ktkostikas@gmail.com

Received: Dec 272014 | Accepted after revision: March 182015 | First published online: April 162015

Conflict of interest: None declared.

Acknowledgements: The results of this study have been presented at the European Respiratory Society Annual Congress in 2013.

\section{References}

1 Mannino DM, Thorn D, Swensen A, et al. Prevalence and outcomes of diabetes, hypertension and cardiovascular disease in COPD. Eur Respir J 2008; 32: 962-969.

2 Dalal AA, Shah M, Lunacsek O, et al. Clinical and economic burden of patients diagnosed with COPD with comorbid cardiovascular disease. Respir Med 2011; 105: 1516-1522.

3 Chakrabarti B, Angus RM, Agarwal S, et al. Hyperglycaemia as a predictor of outcome during non-invasive ventilation in decompensated COPD. Thorax 2009; 64: 857-862.

4 Global Initiative for Chronic Obstructive Lung Disease. Global Strategy for the Diagnosis, Management, and Prevention of Chronic Obstructive Pulmonary Disease. Revised 2011. www.goldcopd.org/uploads/users/files/GOLD_ Report_2011.pdf

5 Global Initiative for Chronic Obstructive Lung Disease. Global Strategy for the Diagnosis, Management and Prevention of Chronic Obstructive Pulmonary Disease. Revised 2013. www.goldcopd.org/uploads/users/files/GOLD_ Report_2013.pdf

6 Lange P, Marott JL, Vestbo J, et al. Prediction of the clinical course of chronic obstructive pulmonary disease, using the new GOLD classification: a study of the general population. Am J Respir Crit Care Med 2012; 186: 975-981.

7 Jones PW, Harding G, Berry P, et al. Development and first validation of the COPD Assessment Test. Eur Respir J 2009; 34: 648-654.

$8 \quad$ Mahler DA, Wells CK. Evaluation of clinical methods for rating dyspnea. Chest 1988; 93: 580-586.

9 World Health Organization Regional Office for Europe. Definition of cardiovascular diseases. www.euro.who.int/ en/what-we-do/health-topics/noncommunicable-diseases/cardiovascular-diseases/definition

10 Hurst JR, Vestbo J, Anzueto A, et al. Susceptibility to exacerbation in chronic obstructive pulmonary disease. N Engl J Med 2010; 363: 1128-1138.

11 Sin DD, Man SF. Chronic obstructive pulmonary disease: a novel risk factor for cardiovascular disease. Can J Physiol Pharmacol 2005; 83: 8-13.

12 Curkendall SM, Lanes S, de Luise C, et al. Chronic obstructive pulmonary disease severity and cardiovascular outcomes. Eur J Epidemiol 2006; 21: 803-813.

13 Naunheim KS, Wood DE, Krasna MJ, et al. Predictors of operative mortality and cardiopulmonary morbidity in the National Emphysema Treatment Trial. J Thorac Cardiovasc Surg 2006; 131: 43-53.

14 Donaldson GC, Mullerova H, Locantore N, et al. Factors associated with change in exacerbation frequency in COPD. Respir Res 2013; 14: 79.

15 Patel AR, Hurst JR. Extrapulmonary comorbidities in chronic obstructive pulmonary disease: state of the art. Expert Rev Respir Med 2011; 5: 647-662.

16 Suissa S, Dell'Aniello S, Ernst P. Long-term natural history of chronic obstructive pulmonary disease: severe exacerbations and mortality. Thorax 2012; 67: 957-963.

17 Johannessen A, Nilsen RM, Storebo M, et al. Comparison of 2011 and 2007 Global Initiative for Chronic Obstructive Lung Disease guidelines for predicting mortality and hospitalization. Am J Respir Crit Care Med 2013; 188: 51-59.

18 Beghe B, Verduri A, Roca M, et al. Exacerbation of respiratory symptoms in COPD patients may not be exacerbations of COPD. Eur Respir J 2013; 41: 993-995. 